\title{
Topographical Relationships Between the Brainstem Auditory and Somatosensory Evoked Potentials and the Location of Lesions in Posterior Fossa Stroke
}

\author{
Yoshikatsu SHIMbo, Masahiro SAKATA*, Makoto HAYAnO**, \\ and Syuichi MORI**
}

\begin{abstract}
Departments of Neurosurgery and *Dentistry, Itoigawa General Hospital, Itoigawa, Niigata; ${ }^{* *}$ Department of Neurosurgery, Mito Saiseikai General Hospital, Mito, Ibaraki
\end{abstract}

\begin{abstract}
The topographical relationships between the location of brainstem lesions detected by magnetic resonance imaging and abnormality of brainstem auditory evoked potentials (BAEPs) and short-latency somatosensory evoked potentials (SSEPs) were studied in 57 patients with stroke in the posterior fossa. Abnormal BAEPs or SSEPs were associated with lesions involving the pontine tegmentum, and abnormal BAEPs also with lesions at the cerebellar peduncle. Absence of the $\mathrm{V}$ wave in BAEPs and N20 in SSEPs was associated with a localized overlapping area in the pontine tegmentum contralateral to stimulation. The overlapping area associated with loss of $\mathrm{N20}$ coincided with the location of the medial lemniscus. Lesions widely involving the pontine tegmentum caused the disappearance of multiple waves in the BAEPs and SSEPs. Patients who entered prolonged coma or died had total loss of the III, IV, and V waves, bilateral absence to the contralateral response in BAEPs, or loss of N18 in SSEPs. The loss of N18 in SSEPs had a statistically significant correlation with bad outcome, which suggests the superiority of SSEPs for predicting the outcome of stroke and indicates the involvement of some system excluding the medial lemniscus in the generation of N18.
\end{abstract}

Key words: brainstem auditory evoked potentials, short-latency somatosensory evoked potentials, brainstem, stroke, N18, outcome

\section{Introduction}

Lesions in the brainstem cause a variety of abnormalities in the brainstem auditory evoked potentials (BAEPs) and the short-latency somatosensory evoked potentials (SSEPs). Such evoked potentials provide reliable information for predicting the outcome of severe head injuries, ${ }^{2,16)}$ evaluating brain death, and intraoperative monitoring of cranial nerve functions. ${ }^{18)}$ The topographical relationship between the change of BAEPs and SSEPs and the location of lesions has been investigated using computed tomography (CT), ${ }^{5,15,20)}$ magnetic resonance (MR) imaging, ${ }^{6,17)}$ and autopsy findings. ${ }^{7,19,20)}$ These investigations suggest that human BAEPs and SSEPs originate from the anatomical pathways of auditory $^{20)}$ and somatosensory tracts. ${ }^{8)}$ However, the location of generator of each peak of the evoked potentials in the human brainstem remains undeter-

Received November 4, 2002; Accepted February 6, 2003 mined..$^{7,9,12,19,20,25)}$

Stroke affecting the posterior fossa causes a variety of abnormalities in the evoked potentials, and simultaneously causes a well-defined lesion on MR imaging. This study examined 57 cases of stroke with BAEPs and SSEPs, and superimposed the lesions shown by MR imaging on templates to investigate the topographical relationship with the abnormal evoked potentials.

\section{Materials and Methods}

\section{Subjects}

Fifty-seven patients with acute stroke, 42 males and 15 females aged 27 to 86 years (mean 65.5 years), were examined. Stroke was caused by 10 brainstem hemorrhages, two cerebellar hemorrhages, and 45 infarcts due to thrombosis and embolism in the brainstem and/or the cerebellum including nine cases of lateral medullary syndrome. No patient underwent surgery. Subarachnoid hemorrhage and other vascular accidents were excluded. Thirty-one 


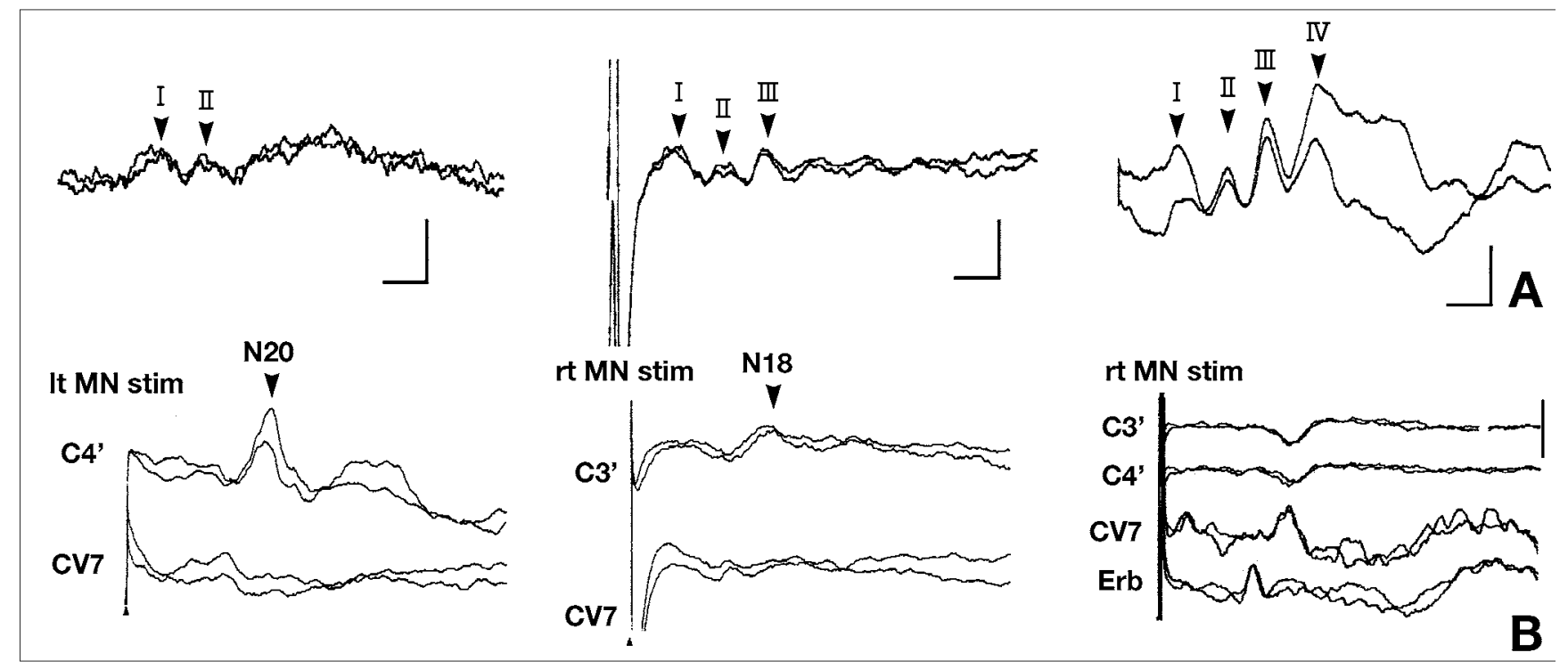

Fig. 1 A: Representative brainstem auditory evoked potential recordings showing the loss of III, IV, and $V$ waves (left), loss of IV and $V$ waves (center), and loss of V wave (right). Identified waves are marked. B: Representative short-latency somatosensory evoked potential recordings showing normal pattern (left), loss of N20 (center), and loss of N18 (right). Reference electrodes were placed on both earlobes. Recording electrodes are shown with stimulation side of the median nerve $(\mathrm{MN})$.

patients were examined at Mito Saiseikai General Hospital from November 1993 through October 1995, and 26 patients at Itoigawa General Hospital from November 1995 through July 2000.

\section{Evoked potentials}

BAEPs and SSEPs were measured in 55 patients and 53 patients, respectively, at one day (day 1) to 64 days (day 64) after onset (mean 11.4 days): 29 cases at 1-7 days, 14 cases at 8-14 days, 10 cases at 15-30 days, and four cases at 30-64 days. Records from patients who suffered brain death were not included in this study. Some patients underwent a second examination (25 cases at 9-280 days) and third examination (7 cases at 65-513 days). SSEPs and BAEPs were examined with a Neuropack II (Nihon Kohden, Tokyo) at Mito Saiseikai General Hospital and a Synax 1100 (NEC San-ei, Tokyo) at Itoigawa General Hospital.

BAEPs were obtained by monaural stimulation using a rarefaction click sound of $85 \mathrm{~dB}$ at $10 \mathrm{~Hz}$. Recording electrodes were placed on both earlobes and a reference electrode was placed at scalp $\mathrm{Cz}$ of the $10-20$ system. The recording period was $10 \mathrm{msec}$, and evoked potentials were amplified and filtered between 50 and $3000 \mathrm{~Hz}$. Totals of 1000 to 2000 responses were averaged. The reproducibility of waveforms was confirmed by repeating trials.
Results were obtained from records ipsilateral to stimulation (ipsilateral lead: Ai), such as Cz-A1 after stimulation of the left ear. Abnormality was determined by the following criteria: loss of any wave $\mathrm{I}-\mathrm{V}$, prolongation over $3 \mathrm{SD}$ of the latency of any wave, or prolongation over 3 SD of the interpeak latency. The normal value of latencies of the Mito Saiseikai General Hospital was obtained from 20 healthy subjects. The normal value at the Itoigawa General Hospital was obtained from the literature. ${ }^{11)}$ Representative recordings are shown in Fig. 1A. The waveforms obtained in the contralateral lead (Ac) were judged by the presence or absence of definite peaks. For example, a case without a reproducible peak in the $\mathrm{Cz}-\mathrm{A} 2$ lead after stimulation of the left ear was judged as a negative response.

SSEPs were recorded after unilateral electrical stimulation of the median nerve in the wrist at $4.5 \mathrm{~Hz}$ with a current of supra-maximal threshold triggering twitches of the thumb. The recording electrodes of the Neuropack II were placed at C3' or C4' (2 cm posterior to C3 or C4 of the 10-20 system) and the neck over the seventh cervical process (CV7). The recording electrodes of the Synax 1100 were placed on C3', C4', CV7, and the ipsilateral Erb' point (Erb). Reference electrodes were placed on both earlobes, which were linked (A1 + A2). The recording period was up to 40 or $50 \mathrm{msec}$, and 
Table 1A Evoked potentials in patients who became communicative or entered locked-in syndrome

\begin{tabular}{|c|c|c|c|c|c|c|c|c|c|c|c|c|c|c|c|c|c|c|c|}
\hline \multirow{3}{*}{$\begin{array}{c}\text { Case } \\
\text { No. }\end{array}$} & \multicolumn{12}{|c|}{ BAEPs } & \multicolumn{6}{|c|}{ SSEPs } & \multirow{3}{*}{ Outcome } \\
\hline & \multicolumn{5}{|c|}{ A1STM } & \multicolumn{5}{|c|}{ A2STM } & \multirow{2}{*}{$\begin{array}{l}\text { A1 } \\
\text { Ac }\end{array}$} & \multirow{2}{*}{$\begin{array}{l}\mathrm{A} 2 \\
\mathrm{Ac}\end{array}$} & \multicolumn{3}{|c|}{ C3' } & \multicolumn{3}{|c|}{ C4' } & \\
\hline & $\mathrm{V}$ & IV & III & II & I & V & IV & III & II & I & & & N20 & N18 & $\mathrm{P} 13 / 14$ & N20 & N18 & $\mathrm{P} 13 / 14$ & \\
\hline 1 & - & - & - & + & + & - & - & - & - & + & $x$ & $x$ & + & + & + & + & + & + & dead \\
\hline 2 & + & + & - & - & - & + & + & + & + & + & $\bigcirc$ & $\bigcirc$ & + & + & + & + & + & + & alive \\
\hline 3 & - & - & - & + & + & - & - & - & + & + & $x$ & $\times$ & - & + & + & - & + & + & alive \\
\hline 4 & - & - & + & + & + & - & - & + & + & + & $x$ & $x$ & - & + & + & - & + & + & alive \\
\hline 5 & - & + & + & + & + & + & + & + & + & + & $x$ & 0 & ND & ND & ND & ND & ND & ND & alive \\
\hline
\end{tabular}

Table 1B Evoked potentials in patients who entered prolonged coma or died

\begin{tabular}{|c|c|c|c|c|c|c|c|c|c|c|c|c|c|c|c|c|c|c|c|}
\hline \multirow{3}{*}{$\begin{array}{l}\text { Case } \\
\text { No. }\end{array}$} & \multicolumn{12}{|c|}{ BAEPs } & \multicolumn{6}{|c|}{ SSEPs } & \multirow{3}{*}{ Outcome } \\
\hline & \multicolumn{5}{|c|}{ A1STM } & \multicolumn{5}{|c|}{ A2STM } & \multirow{2}{*}{$\begin{array}{l}\mathrm{A} 1 \\
\mathrm{Ac}\end{array}$} & \multirow{2}{*}{$\begin{array}{l}\mathrm{A} 2 \\
\mathrm{Ac}\end{array}$} & \multicolumn{3}{|c|}{ C3' } & \multicolumn{3}{|c|}{ C4' } & \\
\hline & $\mathrm{V}$ & IV & III & II & I & V & IV & III & II & I & & & N20 & N18 & $\mathrm{P} 13 / 14$ & N20 & N18 & $\mathrm{P} 13 / 14$ & \\
\hline 6 & - & - & - & - & + & - & - & - & - & - & $\times$ & $x$ & - & - & - & - & - & - & dead \\
\hline 7 & - & - & - & + & + & - & - & - & + & + & $\times$ & $\times$ & - & + & + & - & - & + & dead \\
\hline 8 & - & - & - & - & - & - & + & + & + & + & $\times$ & $x$ & - & - & + & - & - & + & dead \\
\hline 9 & - & - & - & + & + & - & - & - & - & + & $\times$ & $x$ & - & - & + & - & + & + & dead \\
\hline 10 & - & - & + & + & + & - & - & + & + & + & $\times$ & $\times$ & - & + & + & - & - & + & dead \\
\hline 11 & - & - & + & + & + & - & + & + & + & + & $\times$ & 0 & - & - & + & - & + & + & dead \\
\hline
\end{tabular}

A1, A2, Ac: contralateral response (Ac) to A1 or A2 stimulation; A1STM: left ear (A1) stimulation; A2STM: right ear (A2) stimulation; BAEPs: brainstem auditory evoked potentials; C3' or C4': leads at $2 \mathrm{~cm}$ posterior to C3 and C4 of the 10-20 system; ND: not done; SSEPs: short-latency somatosensory evoked potentials; - : loss of peak; +: presence of peak; $\bigcirc$ : positive response on Ac lead; $\times$ : negative response on Ac lead.

evoked potentials were amplified and filtered between 10 and $3000 \mathrm{~Hz}$. Totals of 1000 to 1500 responses were averaged. The reproducibility of waveforms was confirmed by repeating trials. Abnormality was assessed by the following criteria: loss of any wave, or prolongation over $3 \mathrm{SD}$ of the N20 latency or of the central conduction time (CCT). Loss of N18 indicates a flat line was seen after a downward notch of P13/14. Loss of N20 means there was no definite overlapping peak on N18 curve. Representative recordings are shown in Fig. 1B. The normal value of the latency of N20 and CCT was obtained from the literature. ${ }^{11)}$

\section{Evoked potentials in comatose patients}

Eleven patients were examined to assess the BAEPs and SSEPs as predictors of the outcome for comatose patients. Five patients were communicative or in locked-in syndrome and six patients suffered prolonged coma or death. Ten patients were examined within 18 days after onset and one patient in a prolonged coma at day 31 . The abnormalities of the BAEPs and SSEPs are shown in Table 1. Statistical correlations between the lost waves in BAEPs or SSEPs and the outcomes were analyzed by the Fisher exact test. Significance was assessed as a $\mathrm{p}$ value of less than 0.05 .

\section{MR imaging}

Toshiba MRT 50-A (0.5 Tesla; Toshiba, Tokyo) scanners were used in the two hospitals. $\mathrm{T}_{1^{-}}$and $\mathrm{T}_{2^{-}}$ weighted images and proton density images were obtained from all patients, except for one patient with basilar artery occlusion and two with pontine hemorrhages who all died. CT was performed in these three cases. MR imaging was routinely taken with serial axial sections of $6 \mathrm{~mm}$ thickness within 2 weeks after stroke.

All images were superposed by the following method. Templates were made of six parallel axial sections of the normal brainstem as follows: The upper medulla oblongata, the lower pons at the pontomedullary junction, the middle pons containing the trigeminal nerves, the upper pons containing the locus ceruleus, the midbrain containing the inferior colliculi, and the hypothalamus containing the superior colliculi of the midbrain. The outlines of the templates were drawn in red using personal computer software (Photoshop; Adobe Systems Inc., San Jose, Calif., U.S.A.). The extent of the lesion in each case on the axial MR images was manually painted onto the corresponding template in translu- 


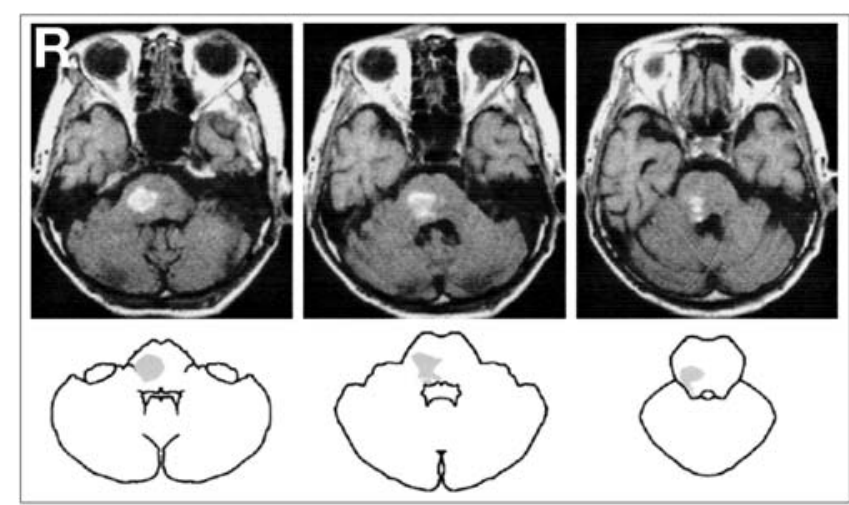

Fig. 2 A 68-year-old man with unilateral pontine hemorrhage. Magnetic resonance images (upper row) demonstrating a hematoma localized in the right tegmentum. The lesions were transferred to our templates of the lower pons, the middle pons, and the upper pons (lower row: left to right, respectively). The left side of all images and diagrams indicates the right side of the brain.

cent blue color. Each case was handled as a layer for superposition. The painting process was based on the anatomical levels of the brainstem and the principal anatomical structures, such as the tegmentum and base, or tectum, tegmentum, and peduncle. The use of axial templates set a limit to representation of the longitudinal ends of lesions. For this reason, the main part of respective lesion was initially painted on suitable templates, and any caudal and rostral ends of the lesion extending to neighboring templates was then drawn. Hematomas were drawn based on high-intensity areas on $\mathrm{T}_{1}$-weighted images, iso- or low-intensity areas on $\mathrm{T}_{2}$-weighted images, or high-density areas on CT. Infarcts were based on high-intensity areas on $\mathrm{T}_{2}$-weighted images. Figure 2 demonstrates an example of a case of pontine hemorrhage. This work was done by one of the authors (Y.S.), who has 9 years' experience in the clinicopathological area of neuropathology. ${ }^{21)}$

Cases were characterized according to the individual pattern of the evoked potentials, and the painted layers were superimposed on each template on the computer screen. As the number of layers increased, the color tone at the overlapping area became denser. In this paper, all figures are reproduced as half tones. The common overlapping area is painted black in the presentation of patterns of loss of certain waves for emphasis. This work was done by one of the authors (M.S.).
Table 2A Results of brainstem auditory evoked potential recordings

\begin{tabular}{llc}
\hline & $\begin{array}{c}\text { Abnormal } \\
\text { pattern }\end{array}$ & $\begin{array}{c}\text { No. of } \\
\text { ears }\end{array}$ \\
\hline Normal group (24 cases) & $\begin{array}{c}\text { - } \\
\text { Abnormal group (31 cases) }\end{array}$ & 0 \\
& II III IV Va & 5 \\
& III IV Va & 4 \\
IV Va & 6 \\
Va & 6 \\
Ip & 5 \\
Vp & 6 \\
I-IIIp & 3 \\
I-Vp & 2 \\
III-Vp & 2 \\
I II IIIa & 2 \\
II III IVa & 1 \\
Ip \& I-Vp & 1 \\
Vre & 1 \\
IV Vre & 1 \\
\hline
\end{tabular}

Table 2B Results of short-latency somatosensory evoked potential recordings

\begin{tabular}{llc}
\hline & $\begin{array}{c}\text { Abnormal } \\
\text { pattern }\end{array}$ & $\begin{array}{c}\text { No. of } \\
\text { nerves }\end{array}$ \\
\hline Normal group (37 cases) & - & 0 \\
Abnormal group (16 cases) & P13/14a & 2 \\
& N18a C3, & 3 \\
& N18a C4' & 3 \\
& N20a C3' & 6 \\
& N20a C4' & 7 \\
& N20p + CCTp & 3 \\
\hline
\end{tabular}

a: loss of wave; C3' or C4': recorded site; CCT: central conduction time; I-III, I-V, and III-V are I-III, I-V, and III-V interpeak latencies, respectively; p: prolongation of latency; re: recovery of wave.

\section{Results}

\section{Evoked potentials and lesion location}

Recording of BAEPs in 55 cases of stroke found no abnormalities in 24 cases and abnormalities in 31 cases. The various abnormal patterns are listed in Table 2A. Representative figures of patterns with lost waves are shown in Fig. 1A. The lesion locations classified by the groups of normal or abnormal BAEPs are demonstrated in Fig. 3. Normal BAEPs were associated with lesions of the medulla oblongata, pontine base, cerebral peduncles, and cerebellar hemispheres. Abnormal BAEPs were apparently associated with lesions involving the pontine tegmentum and cerebellar peduncles. There was no difference in distribution of lesions in the medulla 


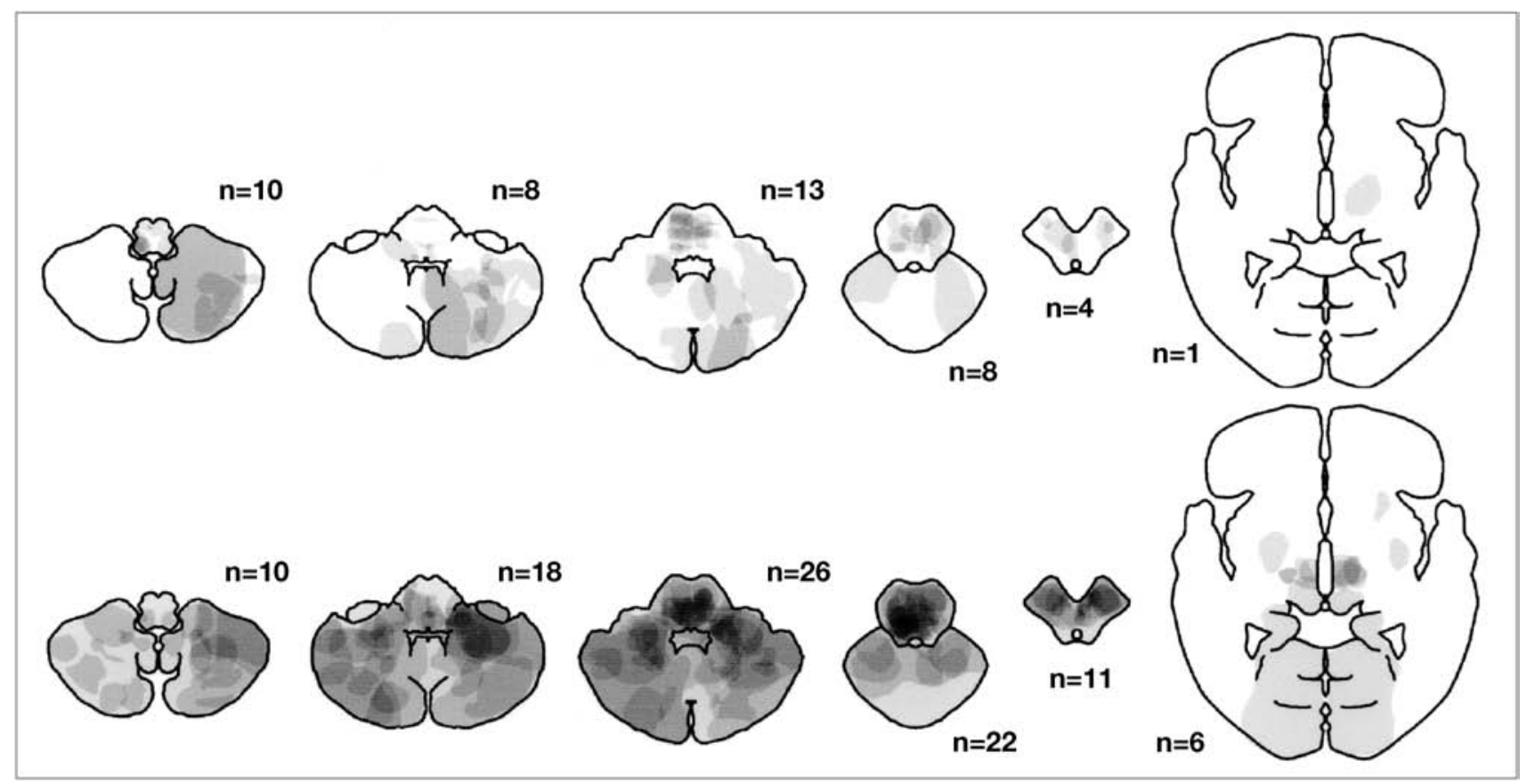

Fig. 3 Templates showing the superimposed lesions of the normal brainstem auditory evoked potential (BAEP) group (upper row) and the abnormal BAEP group (lower row). Medulla oblongata, lower pons, middle pons, upper pons, midbrain, and hypothalamus, from the left to the right. ' $n$ ' shows the number of layers.

oblongata and the midbrain between normal and abnormal groups, suggesting that these two locations are not involved with the formation of BAEPs.

Recordings obtained from the lead contralateral to stimulation (Ac) were studied in the 31 cases of abnormal BAEPs. This contralateral response was assessed as positive or negative. There were three kinds of response as follows: Positive response on both stimulated sides in 16 cases (Fig. 4A), negative response on one side in six cases (Fig. 4B, C), and negative response on both sides in nine cases (Fig. 4D). Lesions in Fig. 4 are restricted to the pons because there was no difference in distribution of lesions in the medulla oblongata and the midbrain. The distribution of negative responses on both sides was associated with lesions widely involving the lower and middle pons (Fig. 4D). On the other hand, the distribution of unilateral negative responses was associated with lesions restricted to the lateral part of the pons or the cerebellar peduncles, indicating malfunction of the entry zone of the auditory pathway (Fig. 4B, C). The latter pattern was also found in the cases without any wave or those with only the I wave as discussed later.

Recording of SSEPs in 53 cases of stroke found no abnormalities in 37 cases and abnormalities in 16 cases. The abnormal patterns are listed in Table 2B.
Representative recordings are demonstrated in Fig. 1B. The distributions of lesions associated with normal and abnormal recordings are demonstrated in Fig. 5, suggesting that lesions of the pontine tegmentum are apparently associated with abnormal SSEPs and lesions of the pontine base and cerebellum are associated with normal SSEPs. There was no difference in distribution of lesions in the medulla oblongata, midbrain, and hypothalamus between normal and abnormal SSEPs. Absence of P13/14 was seen in a case of complete basilar artery occlusion (not shown). The patient was deeply comatose, lacking in brainstem response, and incapable of breathing, but alpha activity was present by conventional electroencephalography. Both C3' and C4' - (A1 + A2) leads showed flat lines and the CV7 - (A1 + A2) lead only recorded N9 and N11 peaks. CT showed a diffusely low-density lesion in the posterior fossa, including the bilateral thalami, as shown in light gray in Fig. 5.

\section{Lesion locations corresponding to specific lost waves}

Total loss of BAEPs without any wave or with only the I wave in eight cases are shown in Fig. 6A. The commonest overlapping lesion area was in the lateral part of the pons or the proximal part of the 


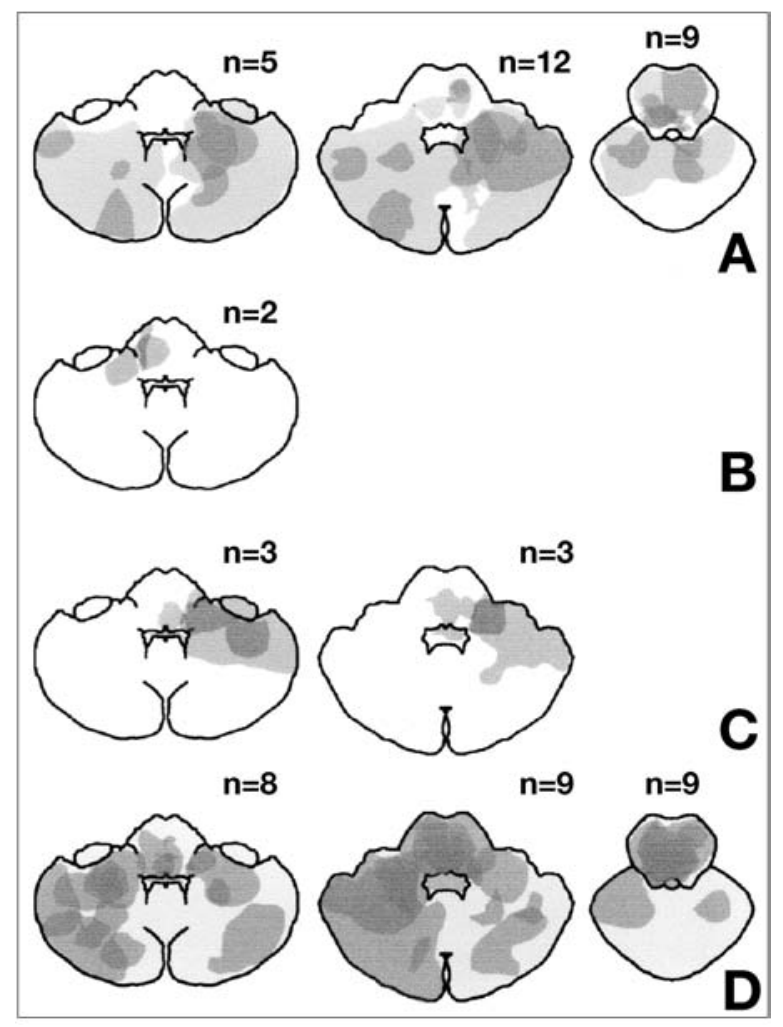

Fig. 4 Templates showing the superimposed lesions of the abnormal brainstem auditory evoked potential group and the contralateral responses. A: Positive response on both sides. B: Negative response to right stimulation. C: Negative response to left stimulation. D: Negative response on both sides. ' $n$ ' shows the number of layers.

cerebellar peduncle ipsilateral to the stimulation, which topographically coincides with the cochlear nucleus.

Loss of III, IV, and V waves occurred in four cases after left ear stimulation (Fig. 6B). Three cases had a wide overlapping lesion area in the tegmentum at the middle and upper pons. The other case had an infarct located in the bilateral cerebellar peduncles but no lesion in the tegmentum of the pons. Two cases of lost III, IV, and V waves occurred after right ear stimulation, which also had a wide lesion area in the pontine tegmentum (not shown).

Loss of IV and V waves occurred in four cases (Fig. 6C), two of which occurred on both sides, and two had unilateral loss of IV and V waves and simultaneous loss of the $\mathrm{V}$ wave on the contralateral side to stimulation. The commonest overlapping lesion area was in the mid-part of the tegmentum of the middle pons for left stimulation and in the right half of the tegmentum of the middle and upper pons for right stimulation.

Loss of the V wave occurred in five cases, after left stimulation in three cases and right stimulation in two cases. The common overlapping lesion area was located at a small part in the right tegmentum of the upper pons, not of the midbrain after left stimulation (Fig. 6D), and in the left tegmentum of the lower and middle pons after right stimulation (not shown).

Loss of N18 of the SSEPs occurred in five cases (Fig. 7A), two cases after right stimulation, two cases after left stimulation, and one case after bilateral stimuli. The common overlapping lesion area was extensively located at the central part of the tegmentum of the middle pons for either stimulation. Additionally, the area extended to the tegmentum of the upper pons after left stimulation, and contained a small part in the tegmentum of the lower pons after right stimulation. The meaning of loss of N18 in this study is no more potential than the electric activity of $\mathrm{A} 1+\mathrm{A} 2$, rather than complete absence of electric activity, because calculation by subtraction of the flat waveform at C3' (C4') - (A1 + A2) lead by the waveform at CV7 - (A1 + A2) lead reveals an apparent negative wave of the latency similar to N18 in the C3' (C4') - CV7 lead.

Loss of N20 occurred in two cases on both sides and in nine cases on one side (Fig. 7B). The common overlapping lesion area was located at a small medial part in the tegmentum of the middle and upper pons on the left side after right stimulation, which topographically coincides with the medial lemniscus. A similar distribution was seen on the right side after left stimulation. There was no overlapping lesion area in the medulla oblongata.

\section{Evoked potentials in comatose patients}

The distribution of the lesions is shown in Fig. 8 and the results of recording of BAEPs and SSEPs are shown in Table 1. Patients suffering prolonged coma or death had large lesions in the middle pons compared with patients in communicative states. A similar distribution of lesions was observed in patients with total loss of III, IV, and V waves in BAEPs, with bilateral absence of the contralateral response of BAEPs, and with loss of N18 in SSEPs. Statistical analysis using the Fisher exact test showed loss of N18 on either or both sides was significantly correlated with prolonged coma or death $(p<0.01)$. The other two patterns of BAEPs were not correlated.

\section{Discussion}

The present study of stroke in the posterior fossa showed that lesions involving the pontine tegmen- 


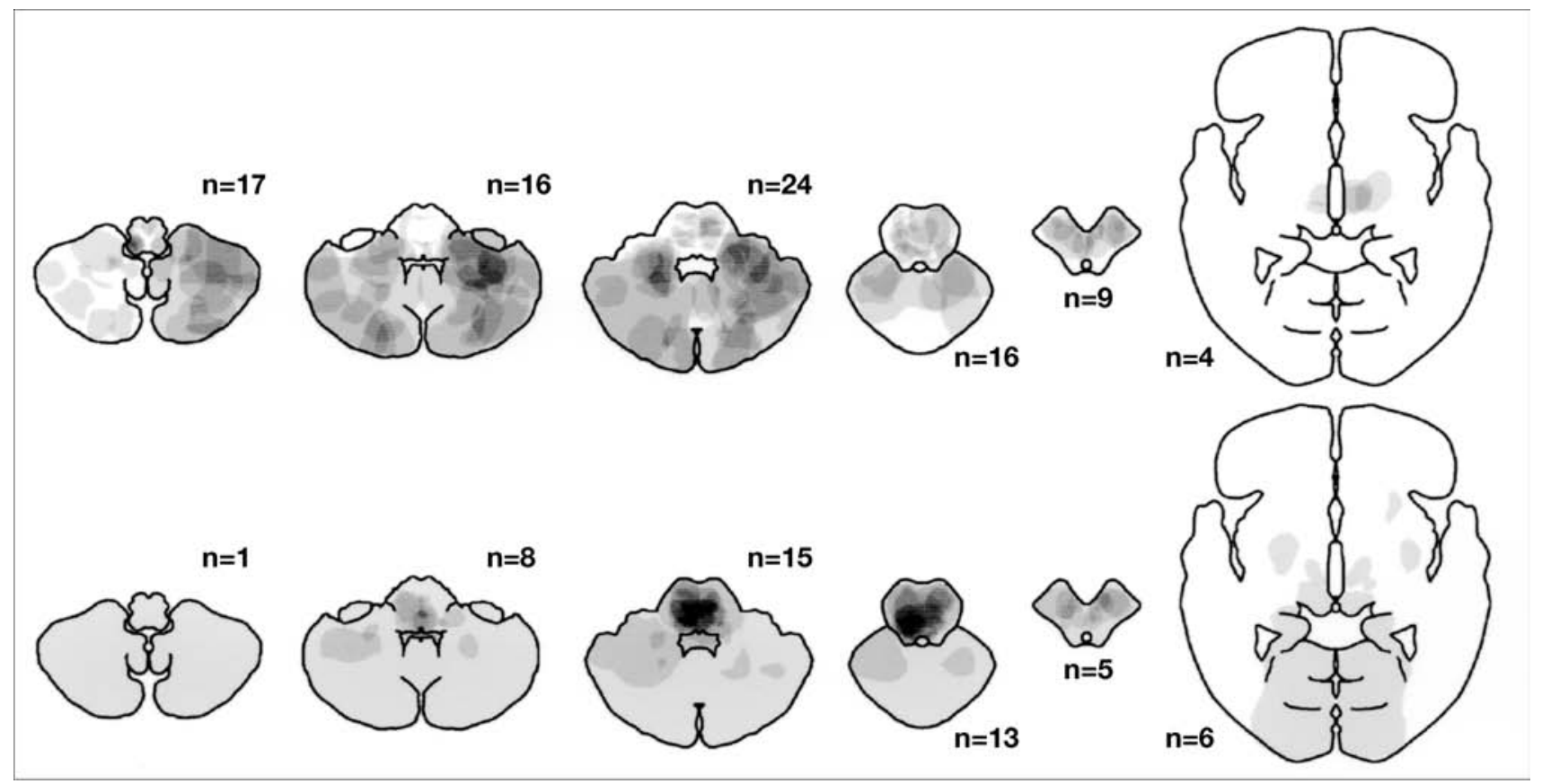

Fig. 5 Templates showing the lesions of the normal short-latency somatosensory evoked potential (SSEP) group (upper row) and abnormal SSEP group (lower row). ' $n$ ' shows the number of layers.
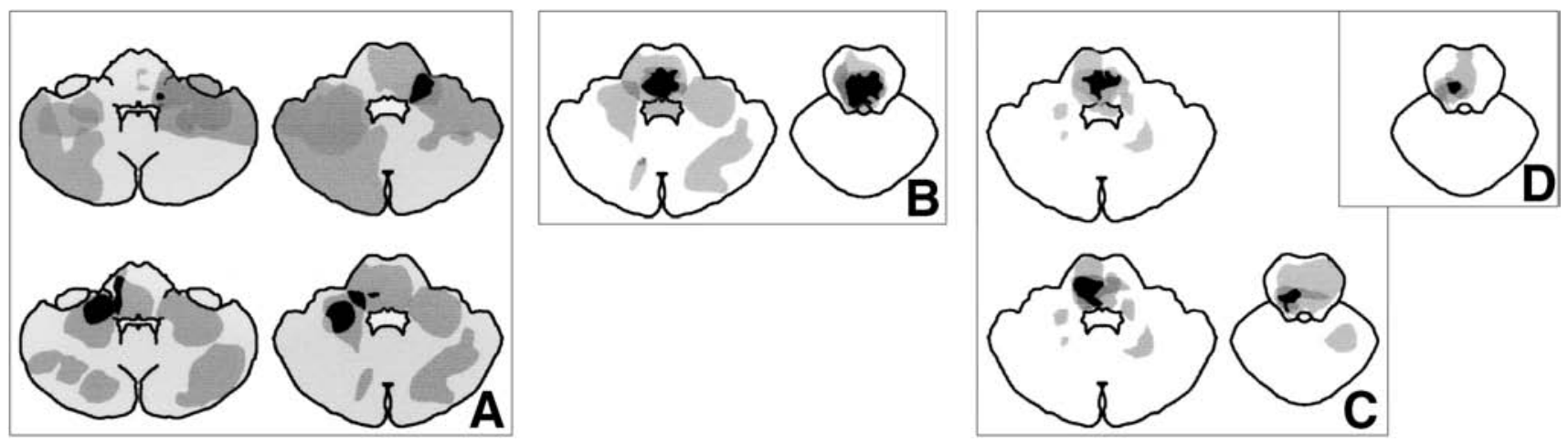

Fig. 6 Templates showing the lesions and individual patterns of loss of waves in brainstem auditory evoked potentials. Solid black areas indicate the common overlapping area in all layers. A: No wave or only I wave by left stimulation (upper, 5 cases) and by right stimulation (lower, 4 cases). B: Loss of III, IV, and V waves by left stimulation (4 cases). C: Loss of IV and V waves by left stimulation (upper, 3 cases) and by right stimulation (lower, 3 cases). D: Loss of $\mathrm{V}$ wave by left stimulation ( 3 cases). Only two cases of loss by right stimulation are not shown in $B$ and $D$.

tum always cause various abnormalities of the BAEPs or SSEPs, lesions at the cerebellar peduncles cause abnormal BAEPs, and lesions in the pontine tegmentum cause loss of the $\mathrm{V}$ wave in BAEPs or loss of N20 in SSEPs. A large lesion involving the bilateral pontine tegmenta causes disappearance of more than one wave in the BAEPs and SSEPs, and may result in prolonged coma or death. The follow- ing patterns are very important for suggesting the presence of fatal large lesions: total loss of III, IV, and V waves in BAEPs; absence of the contralateral response of BAEPs to stimuli on both sides; and loss of N18 in SSEPs. These indications will be helpful to evaluate the prognosis for patients with posterior fossa stroke.

The distribution of lost BAEP waves may be 

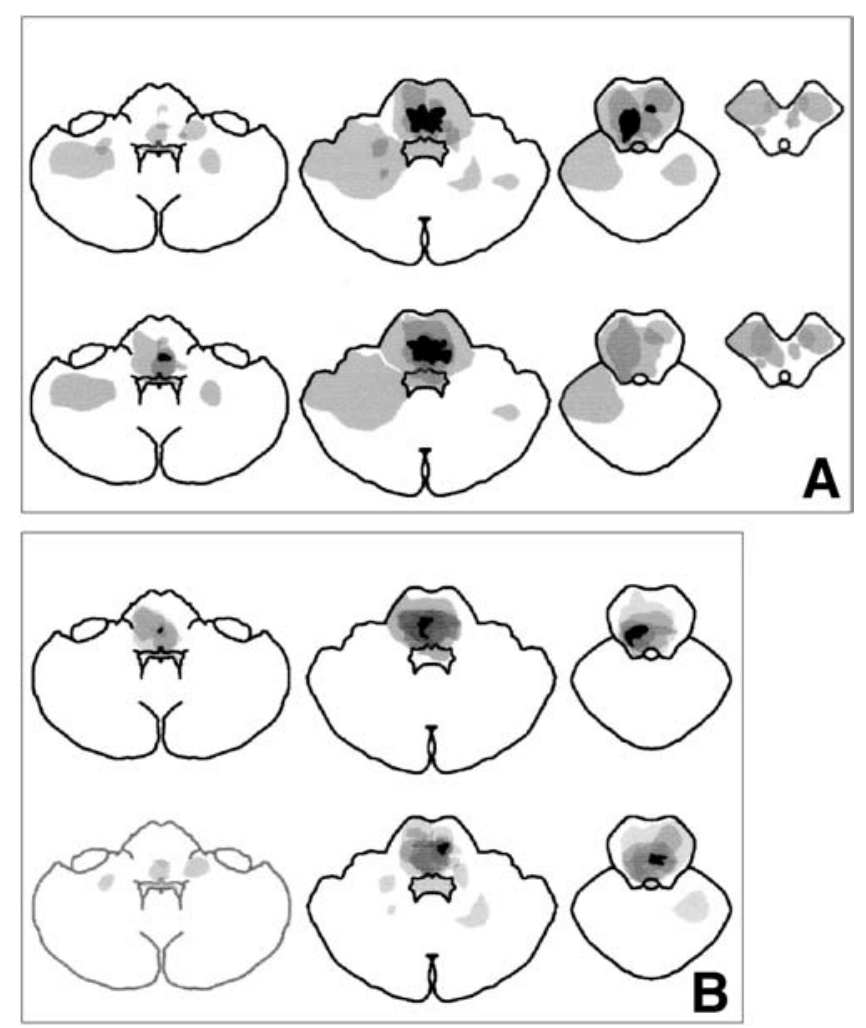

Fig. 7 Templates showing lesions and loss of short-latency somatosensory evoked potential waves. A: Loss of $\mathrm{N} 18$ by left median nerve (MN) stimulation (upper, 3 cases) and by right $\mathrm{MN}$ stimulation (lower, 3 cases). B: Loss of $\mathrm{N20}$ by left MN stimulation (upper, 7 cases) and by right MN stimulation (lower, 6 cases).

closely linked to lost generators of individual waves. The I and II waves are certainly generated near the cochlear nerve and nuclei ipsilateral to stimulation. ${ }^{15,19,20)}$ The distribution of lesions in cases with no waves or only the I wave demonstrated a common overlapping area at the cerebellar peduncles ipsilateral to stimulation. The generators of the waves after the II wave are not known ${ }^{9,15,19,20,23,26)}$ in humans, although the generators of the III and V waves may be related to the superior olivary nucleus $^{1,15)}$ and the inferior colliculus, ${ }^{7)}$ respectively. In this study, total loss of the III, IV, and V waves was associated with an extensive overlapping area occupying the bilateral pontine tegmenta. The overlapping area converged into a localized area as the late component of the waves disappeared. The loss of the $\mathrm{V}$ wave was associated with a small overlapping area in the pontine tegmentum, not in the midbrain, contralateral to stimulation. These findings suggest that auditory impulse processing is integrated inside

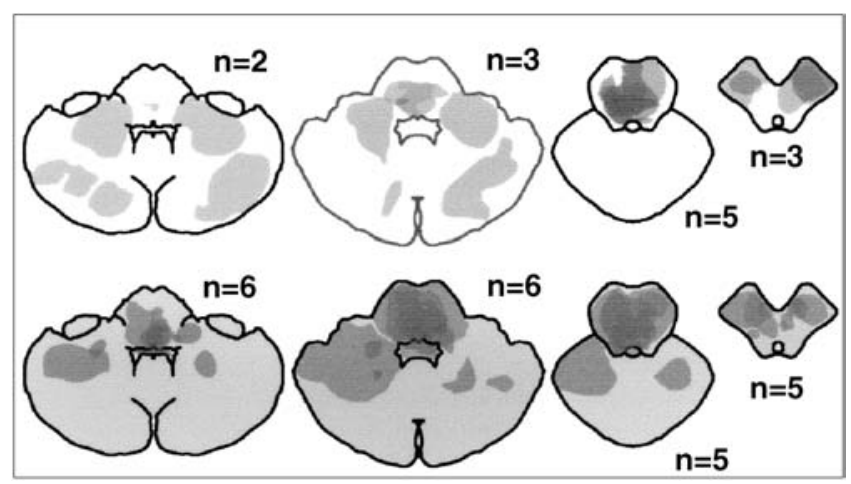

Fig. 8 Templates showing lesions in initially comatose patients. upper row: Five patients who became communicative or entered locked-in syndrome. lower row: Six patients who entered prolonged coma or died, and had large lesions in the middle pons. ' $n$ ' shows the number of layers.

the pons, ${ }^{26)}$ although the topographical localization of the respective generators of the III, IV, and V waves could not be determined. Experimental studies in cats show that each wave of the BAEPs has multiple sources in the auditory brainstem nuclei and tracts, ${ }^{10)}$ and that neuronal loss in the superior olivary complex causes complicated reactions of the late components of the BAEPs. ${ }^{13,27)}$ Our results may suggest that the complicated localization of the generators of the III, IV, and V waves is also present in humans.

The origins of the potentials of the SSEPs have been investigated. ${ }^{12)}$ The $\mathrm{P} 14, \mathrm{~N} 18$, and N20 are believed to be the keys to the intracranial events. The generator of P14 is considered to be located at the nucleus cuneatus. ${ }^{14)}$ Loss of P13/14 was only seen in one case of entire brainstem infarct after complete basilar artery occlusion. N20 is a near field potential delivered from the cortical dipole in the primary sensory cortex contralateral to stimulation.4) The loss of this potential indicates the disappearance of afferent impulses in the levels of the brainstem. In this study, the common overlapping area associated with loss of N20 coincided with the topographical location of the medial lemniscus of the pons in agreement with the anatomy of the somatosensory tract for deep sensation.

N18, a far-field potential, may originate from the thalamus, ${ }^{22)}$ midbrain, ${ }^{24)}$ pons, ${ }^{25)}$ or medulla oblongata. ${ }^{6,17,18)}$ Evidence for the medulla oblongata origin is derived from clinical cases as follows: N18 is preserved if lesions are located at the pontine medial lemniscus, whereas a lesion at $\mathrm{C} 1-\mathrm{C} 2$ results in the absence of N18. ${ }^{17)}$ Tumor extension caudally to the 
medulla oblongata results in the disappearance of N18. ${ }^{6)}$ Complete loss of N18 is only found if brain death occurs, suggesting that the generator of N18 may be the medulla oblongata. ${ }^{18)}$ On the other hand, evidence for the origin in the pons or midbrain is derived from intracranial recording as follows: The latency of the negative potential initially recorded at the medulla oblongata gradually increased with movement of the electrode from the medulla oblongata to the upper pons or the midbrain, and finally became a fixed potential at the upper brainstem..$^{8,25)}$ This potential may reflect an ascending axonal potential along the medial lemniscal pathway. ${ }^{14)}$ Therefore, the N18 potential may be generated on the medial lemniscus pathway.

Loss of N18 in our cases reflected a marked decrease in potential equal to A1 + A2 and has indicated the existence of a large lesion involving the bilateral pontine tegmenta, regardless of the stimulation site. Extensive lesions in the pontine tegmentum were also associated with loss of the III, IV, and $\mathrm{V}$ waves and bilateral absence of the contralateral responses in BAEPs. Our study of comatose patients showed a statistically significant correlation between loss of N18 and prolonged coma or death. This result suggests the superiority of SSEPs for predicting the outcome of brainstem stroke and the close relationship between $\mathrm{N} 18$ and the arousal system, that is, the reticular formation of the brainstem. Some extralemniscal tracts, like the anterior spinothalamic tract, pass through the medial lemniscus and provide collateral projections to the reticular formation, or the spinoreticular tract, which terminates at the bilateral pontine reticular formation. ${ }^{3)}$ We assume that N18 is generated in collateral projections to the reticular formation from some type of extralemniscal tracts, chiefly in the pons. Further research in humans and careful experiments with special animal models may confirm this assumption.

\section{References}

1) Buchwald JS, Huang C-M: Far-field acoustic response: origins in the cat. Science 189: 382-384, 1975

2) Cant BR, Hume AL, Judson JA, Shaw NA: The assessment of severe head injury by short-latency somatosensory and brain-stem auditory evoked potentials. Electroencephalogr Clin Neurophysiol 65: 188-195, 1986

3) Carpenter MB: Human Neuroanatomy, ed 7. Baltimore, Williams \& Wilkins, 1976, pp 388-393

4) Dinner DS, Lüders H, Lesser RP, Morris HH: Cortical generators of somatosensory evoked potentials to median nerve stimulation. Neurology 37: 1141-1145,
1987

5) Ferbert A, Buchner H, Brockmann H: Brainstem auditory evoked potentials and somatosensory evoked potentials in pontine haemorrhage. Correlations with clinical and CT findings. Brain 113: 49-63, 1990

6) Fukuda M, Kameyama S, Honda Y, Yamazaki H, Kawaguchi T, Tamura A, Suzuki K, Tanaka R: Shortlatency somatosensory evoked potentials in patients with brain stem tumor: Study of N20 and N18 potentials. Neurol Med Chir (Tokyo) 37: 525-532, 1997

7) Hammond EJ, Wilder BJ, Goodman IJ, Hunter SB: Auditory brain-stem potentials with unilateral pontine hemorrhage. Arch Neurol 42: 767-768, 1985

8) Hashimoto I: Somatosensory evoked potentials from the human brain-stem: origins of short latency potentials. Electroencephalogr Clin Neurophysiol 57: 221-227, 1984

9) Hashimoto I, Ishiyama Y, Yoshimoto T, Nemoto S: Brain-stem auditory-evoked potentials recorded directly from human brain-stem and thalamus. Brain 104: 841-859, 1981

10) Kaga K, Shinoda Y, Suzuki JI: Origin of auditory brainstem responses in cats: whole brainstem mapping, and a lesion and HRP study of the inferior colliculus. Acta Otolaryngol 117: 197-201, 1997

11) Kameyama S: [Electrophysiological Monitoring for Neurosurgical Operations]. Niigata, Nishimura, 1997, pp 26-34 (Jpn)

12) Lee EK, Seyal M: Generators of short latency human somatosensory-evoked potentials recorded over the spine and scalp. J Clin Neurophysiol 15: 227-234, 1998

13) Melcher JR, Guinan JJ Jr, Knudson IM, Kiang NY: Generators of the brainstem auditory evoked potential in cat. II. Correlating lesion sites with waveform changes. Hear Res 93(1-2): 28-51, 1996

14) Morioka T, Tobimatsu S, Fujii K, Fukui M, Kato M, Matsubara T: Origin and distribution of brain-stem somatosensory evoked potentials in humans. Electroencephalogr Clin Neurophysiol 80: 221-227, 1991

15) Oh SJ, Kuba T, Soyer A, Choi IS, Bonikowski FP, Vitek J: Lateralization of brainstem lesions by brainstem auditory evoked potentials. Neurology 31: 14-18, 1981

16) Soldner F, Holper BM, Chone L, Wallenfang $\mathrm{T}$ : Evoked potentials in acute head injured patients with MRI-detected intracerebral lesions. Acta Neurochir (Wien) 143: 873-883, 2001

17) Sonoo M, Genba K, Zai W, Iwata $M$, Mannen $T$, Kanazawa I: Origin of the widespread N18 in median nerve SEP. Electroencephalogr Clin Neurophysiol 84: 418-425, 1992

18) Sonoo M, Tsai-Shozawa $Y$, Aoki $M$, Nakatani $T$, Hatanaka Y, Mochizuki A, Sawada M, Kobayashi K, Shimizu T: N18 in median somatosensory evoked potentials: a new indicator of medullary function useful for the diagnosis of brain death. J Neurol Neurosurg Psychiatry 67: 374-378, 1999

19) Starr A, Hamilton AE: Correlation between confirmed sites of neurological lesions and abnormali- 
ties of far-field auditory brainstem responses. Electroencephalogr Clin Neurophysiol 41: 595-608, 1976

20) Stockard JJ, Rossiter VS: Clinical and pathologic correlates of brain stem auditory response abnormalities. Neurology 27: 316-325, 1977

21) Tsuchida T, Shimbo Y, Fukuda M, Takeda N, Tanaka R, Ikuta F: Computed tomographic and histopathological studies of pontine glioma. Childs Nerv Syst 1: 223-229, 1985

22) Tsuji S, Shibasaki H, Kato M, Kuroiwa Y, Shima F: Subcortical, thalamic and cortical somatosensory evoked potentials to median nerve stimulation. Electroencephalogr Clin Neurophysiol 59: 465-476, 1984

23) Urasaki E, Uematsu S, Lesser RP: Brainstem auditory evoked potentials recorded around the human upper brainstem area. Neurol Med Chir (Tokyo) 35: 353-359, 1995

24) Urasaki E, Wada S, Kadoya C, Tokimura T, Yokota A, Yamamoto S, Fukumura A, Hamada S: Amplitude abnormalities in the scalp far-field N18 of SSEPs to median nerve stimulation in patients with midbrainpontine lesion. Electroencephalogr Clin Neurophysiol 84: 232-242, 1992
25) Urasaki E, Wada S, Kadoya K, Yokota A, Matsuoka S, Shima F: Origin of scalp far-field N18 of SSEPs in response to median nerve stimulation. Electroencephalogr Clin Neurophysiol 77: 39-51, 1990

26) Vitte E, Tankere F, Bernat I, Zouaoui A, Lamas G, Soudant J: Midbrain deafness with normal brainstem auditory evoked potentials. Neurology 58: 970-973, 2002

27) Zaaroor M, Starr A: Auditory brain-stem evoked potentials in cat after kainic acid induced neuronal loss. I. Superior olivary complex. Electroencephalogr Clin Neurophysiol 80: 422-435, 1991

Address reprint requests to: Y. Shimbo, M.D., Department of Neurosurgery, Itoigawa General Hospital, 457-1 Takegahana, Itoigawa, Niigata 941-8502, Japan. e-mail: yoshi.00@poem.ocn.ne.jp.

Commentary on this paper appears on the next page. 


\section{Commentary}

Shimbo and colleagues have made a substantive contribution to our understanding to the topographic substrate underlying brainstem auditory and somatosensory evoked potentials. Improvements in neuroimaging, and particularly in MRI, enable in vivo categorization of brainstem stroke. Although diffusionweighted sequences were not part of this study, T1and T2-weighted images, proton density images as well as CT images (to what respective degree is not discernible in the data) provided sufficient identification of stroke localization to effect correlation with abnormalities in these evoked responses.

The correlation methodology adopted for this study is based in logic, rigorous and powerful. The spatial co-registration presumably is reasonably valid; brainstem variability is certainly less than that of the telencephalon. Intuitively, this might be more legitimate in ischemic than in hemorrhagic stroke, given the greater likelihood of displacement of functional tissue in the latter. Further analysis of the data for this purpose might add meaningful insight to this assumption.

The most interesting correlation finding of this study may be that pertaining to wave $V$ of the BAER and the pontine tegmentum, but as the authors note, $a$ simplistic correlation of each and every wave component with an anatomic structure has never been expected or thought very likely. Separate from their primary objective, the authors found a statistical correlation between loss of N18 in the SSEP and a poor clinical outcome, but it is not evident that a possible correlation between imaging findings and clinical outcome was investigated. Given the attempt to correlate imaging with electrophysiology and given the study's clever methodology to investigate such a correlation, this would have been a most interesting, although recognizably much more difficult, question to address.
David W. RoberTs, M.D.

Section of Neurosurgery

Dartmouth-Hitchcock Medical Center Lebanon, New Hampshire, U.S.A.

Shimbo and co-workers described a topographical relationship between the brainstem auditory evoked potentials (BAEPs) and short latency somatosensoryevoked potentials (SSEPs), and the location of lesions in their series of patients with posterior fossa stroke. In the present study, 12 cases of hemorrhage and 45 cases of infarction were subjected to the examination. The authors clearly demonstrated the characteristics of the BAEPs and SSEPs in patients with a large lesion involving the bilateral pontine tegmenta. Total loss of III, IV and V waves in BAEPs, absence of the contralateral response of BAEPs to stimuli on both sides, and loss of N18 in SSEPs are the most critical findings in this study. These results were well correlated with the fatal outcome of the patients.

However, the recordings of these evoked potentials were performed from the first day to 64th day after the insult of the posterior stroke. Under these conditions, recorded evoked potentials were quite different in neurophysiological state from the acute to chronic phases. The authors performed a second study in 25 patients and a third study in 7 patients. But there is no description or analysis of the evoked potential examination of the follow-up study. For the next step, the authors should examine the follow-up BAEPs and SSEPs to determine whether it is useful to indicate the possible recovery of the patients from posterior fossa stroke using these evoked potential studies.

Tatsuya TANAKA, M.D. Department of Neurosurgery Asahikawa Medical College Asahikawa, Hokkaido, Japan 